\title{
Solar neutrinos: status and prospects
}

\author{
Marianne Göger-Neff* \\ Technische Universität München \\ E-mail: marianne.goeger@ph.tum.de
}

The Sun is an ideal laboratory for the study of fundamental neutrino properties, as well as neutrinos are unique messengers from the solar core. Over the last forty years, the study of solar neutrinos has significantly contributed to progress in both astrophysics and elementary particle physics. After the establishment of neutrino oscillations as the solution of the Solar Neutrino Problem, the focus of current solar neutrino experiments lies on the improvement of the astrophysical model of the Solar interior, the standard Solar Model. Open questions, like the vacuum-to-matter transition of the solar neutrino oscillations, or the solar metallicity problem, can be addressed by a precise determination of the solar neutrino fluxes in the low and intermediate energy region $(<4 \mathrm{MeV})$. In this context, I will summarize the recent results from SNO, SuperKamiokande and Borexino.

XIII Nuclei in the Cosmos,

7-11 July, 2014

Debrecen, Hungary

\footnotetext{
* Speaker.
} 


\section{Introduction}

Electron neutrinos are constantly produced in our Sun in the process of hydrogen burning: $4 p \rightarrow{ }^{4} \mathrm{He}+2 e^{+}+2 v_{e} .99 \%$ of the reactions go through the so-called $p p$ chain while only $\sim 1 \%$ go through the $C N O$ cycle, a process which is dominant in heavier stars. These solar neutrinos offer the unique possibility to study at the same time fundamental particle properties as well as to reveal information about the solar interior. The predicted solar neutrino fluxes and spectra from the individual reactions of the pp chain and CNO cycle are depicted in fig.1.

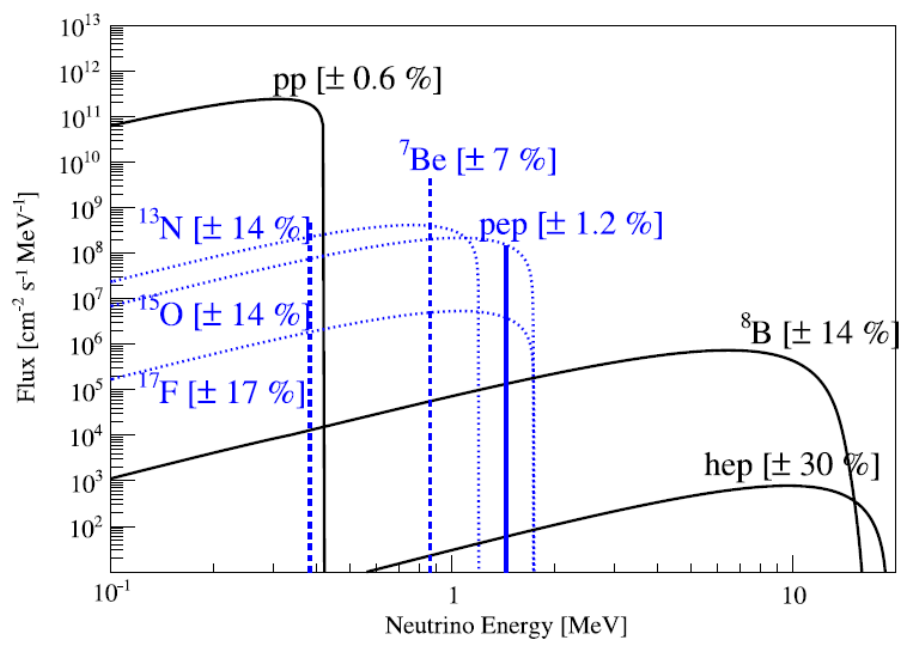

Figure 1: The solar neutrino spectrum as predicted by the standard solar model (assuming high metallicity), including theoretical uncertainties.

Getting information about the solar core and thus confirming the theory of nuclear burning in stars was the motivation for the first solar neutrino experiment suggested in the 1960s by J.N Bahcall and R. Davis Jr., the Chlorine experiment $[1,2]$. But this pioneering experiment as well as its successors - Kamiokande, Gallex/GNO, Sage and Super-Kamiokande - measured a significantly lower neutrino flux than predicted, leading to the Solar Neutrino Problem. The proposed solutions either questioned the astrophysical model of the solar interior, the Standard Solar Model (SSM) or included unknown neutrino properties as neutrino flavor oscillations, neutrino magnetic moment or neutrino decay. After more than 30 years the Solar Neutrino Problem was finally solved by the SNO experiment in 2002, which could prove that the full flux of ${ }^{8} \mathrm{~B}$ neutrinos is present in the neutral-current-channel, while $2 / 3$ of the electron neutrino flux is missing. This means that $\sim 2 / 3$ of the electron neutrinos created in the Sun are transformed in other neutrino flavors on their way to detection via matter enhanced resonant conversion, the so-called MSW-effect. Lower energy $(<2 \mathrm{MeV})$ solar neutrino data are not affected by the matter resonance and are best described as averaged vacuum oscillations. A combined analysis of all solar neutrino experiments together with the reactor neutrino experiment KamLAND leads to the present best fit values for the oscillation parameters $\sin ^{2} 2 \theta_{12}=0.846 \pm 0.021, \Delta m_{21}^{2}=(7.53 \pm 0.18) \cdot 10^{-5} \mathrm{eV}^{2}$ [3] which is known as large mixing angle (LMA) solution. During the last decade, the experimental efforts have focused on the detection of low energy solar neutrinos in order to confirm the oscillation model also in an energy region where vacuum oscillations dominate, and to confirm the standard solar model via the direct 
detection of the much more abundant low energetic neutrino branches, namely the ${ }^{7} \mathrm{Be}$, pep and pp neutrinos.

In the following I will summarize the recent results from the two still running solar neutrino experiments, Borexino and Super-Kamiokande, and from a reanalysis of the low energy data of the SNO experiment which stopped data taking in 2006.

\section{Recent Solar Neutrino Results}

\subsection{SNO}

The SNO detector located in the Sudbury mine, Canada, used $1000 \mathrm{t}$ of heavy water $\left(\mathrm{D}_{2} \mathrm{O}\right)$ as target. The Cherenkov light created by charged particles was detected by 9500 PMTs. Solar neutrinos could be detected via three different reactions, namely a charged-current reaction (CC) possible only for electron neutrinos, a neutral-current interaction (NC) possible for neutrinos of all flavors and neutrino electron scattering (ES), which is possible for all neutrino flavors, but with an about 6 times higher cross section for electron neutrinos than the other flavors. The comparison of these different reaction channels in one detector provided the unambiguous proof of neutrino oscillations.

The SNO collaboration published their final results in 2013, including an analysis with the detection threshold lowered from $5.0 \mathrm{MeV}$ to $3.5 \mathrm{MeV}$ [4]. The measured ${ }^{8} \mathrm{~B}$ neutrino flux is $\left(5.25 \pm 0.16_{\text {stat }} \pm 0.13_{\text {syst }}\right) \cdot 10^{6} \mathrm{~cm}^{-2} \mathrm{~s}^{-1}$. The electron neutrino survival probability together with the low energy threshold ${ }^{8} \mathrm{~B}$ neutrino spectrum measured by SNO is shown in fig. 2. The best estimate of the survival probability between 4 and $9 \mathrm{MeV}$ seems to be in disagreement with the expectation (though compatible at $1 \sigma$ level), showing a lower survival probability for lower energies, while the opposite behavior is expected for the LMA solution. Better data in this energy region are needed in order to prove or disprove this result.

\subsection{Super-Kamiokande}

Super-Kamiokande is a $40 \mathrm{~m}$ diameter, $40 \mathrm{~m}$ tall cylindrical stainless steel tank filled with 50 ktons of ultrapure water, located in the Kamioka mine, Japan. The Cherenkov light created by charged particles in the water is detected by 11000 PMTs. The experiment is running since 1996 and has accumulated by far the highest statistics of ${ }^{8}$ B neutrinos. Super-Kamiokande detects solar neutrinos via neutrino-electron scattering (ES), and is thus sensitive also to other flavors than electron neutrinos though with a reduced cross section. In the phase IV of the experiment, starting in 2008 , the detection threshold could be lowered to $3.5 \mathrm{MeV}$, thanks to the successful reduction of the Radon background.

Super-Kamiokande measures an electron equivalent ${ }^{8} \mathrm{~B}$ neutrino flux of $\Phi_{8_{B}}=\left(2.37 \pm 0.015_{\text {stat }} \pm\right.$ $\left.0.04_{\text {syst }}\right) \cdot 10^{6} \mathrm{~cm}^{-2} \mathrm{~s}^{-1}$ thus confirming that about $2 / 3$ of the electron neutrinos are converted into other flavors.

The low threshold detection of the ${ }^{8} \mathrm{~B}$ neutrino flux had the goal to verify the low energy upturn in the spectrum, which is expected as a consequence of the higher survival probability at lower energies for the MSW-LMA solution. While in earlier phases of the experiment, the upturn could not be proven, the experimental spectrum above $3.5 \mathrm{MeV}$ now is compatible with distortions due 


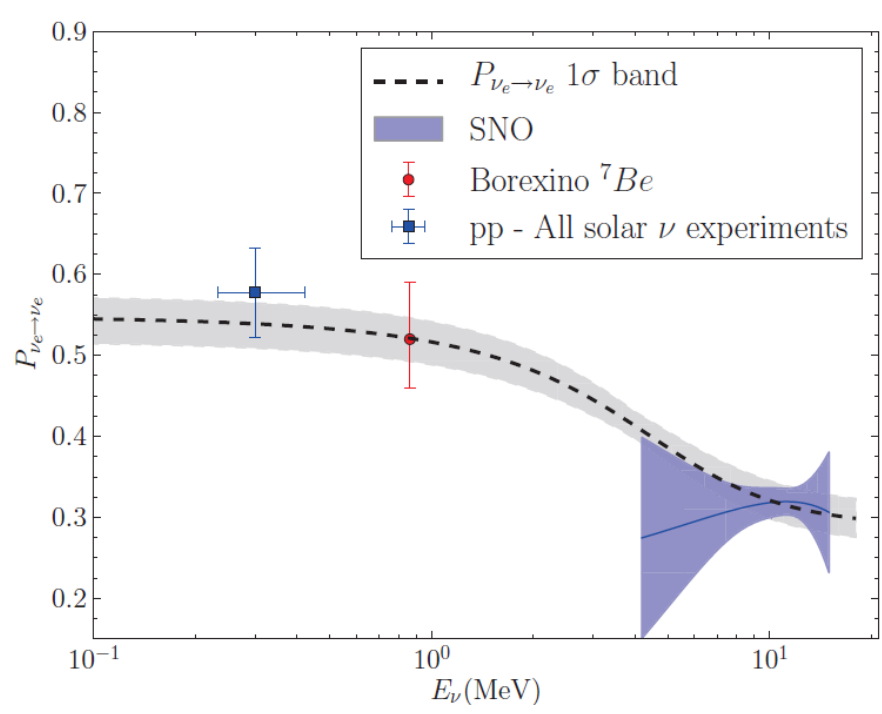

Figure 2: The predicted electron neutrino survival probability for the best-fit LMA solution is depicted with the dashed black line and the errors indicated by the grey band, while the measured survival probability for the low energy ${ }^{8} \mathrm{~B}$ spectrum measured by SNO is depicted in blue, and the one for ${ }^{7} \mathrm{Be}$ neutrinos measured by Borexino is plotted in red. Taken from [4].

to neutrino oscillations [6]. More statistics and a further reduction of the detection threshold might improve the situation in the future.

Due to the real-time detection of the solar neutrinos, Super-Kamiokande can also search for a day-night-asymmetry in the neutrino flux, an effect which can be explained by earth matter effects affecting the solar neutrinos during the night (when the Sun is below the horizon). SuperKamiokande measures a small asysmmetry of $A_{D N}=\left(-3.2 \pm 1.1_{\text {stat }} \pm 0.5_{\text {sys }}\right) \%$ which is in very good agreement with the expected value of $3.3 \%$ for the MSW effect [5].

\subsection{Borexino}

The Borexino experiment has been running since May 2007 at the Gran Sasso underground laboratory in Italy with the primary goal of studying low-energy solar neutrinos in real-time. The detecor consists of an unsegmented volume of $300 \mathrm{t}$ of ultrapure liquid scintillator, contained in a nylon balloon of $8.5 \mathrm{~m}$ diameter and a thickness of $125 \mu \mathrm{m}$ immersed in $1000 \mathrm{t}$ of organic buffer liquid. The scintillation light is detected by 2200 PMTs. Solar neutrinos are detected in Borexino through their elastic scattering on electrons in the scintillator. The high light yield of the liquid scintillator together with a very low background level allowed the first real-time detection of solar neutrinos below $1 \mathrm{MeV}$.

During the Phase I (2007 - 2010), Borexino made the first direct measurements of ${ }^{7} \mathrm{Be},{ }^{8} \mathrm{~B}$ and pep neutrinos, and placed a limit on the CNO neutrino flux. In the 2 nd phase of the experiment which has started 2011, Borexino aims now to measure the major pp neutrino flux and to improve the limits on the CNO neutrino flux: in this way it will become the first experiment able to perform a complete spectroscopy of the whole solar neutrino flux. 


\section{Precision measurement of ${ }^{7}$ Be neutrinos}

The latest ${ }^{7} \mathrm{Be}$ result has been published in [7]. Taking into account the systematic errors, stemming essentially from the uncertainty in the energy scale and in the fiducial volume selection, the measured ${ }^{7} \mathrm{Be}$ rate is $\left(46 \pm 1.5_{\text {stat }} \pm 1.6_{\text {syst }}\right) \mathrm{cpd} / 100$ tons. Under the assumption of neutrino oscillations this corresponds to a total flux of $\Phi_{7_{B e}}=(4.84 \pm 0.24) \cdot 10^{9} \mathrm{~cm}^{-2} \mathrm{~s}^{-1}$ which is in good agreement with the prediction of the Standard Solar Model. The combined error is less than 5\%, which is smaller than the theoretical uncertainty of the flux prediction.

\section{Low energy ${ }^{8}$ B spectrum}

The measurement of the ${ }^{8} \mathrm{~B}$ neutrino flux performed by Borexino has with $3 \mathrm{MeV}$ a significantly lower threshold than the previous measurements from the Cherenkov experiments, but suffers from a lower statistics. The observed ${ }^{8} \mathrm{~B}$ rate in the detector is $\left(0.217 \pm 0.038_{\text {stat }} \pm 0.008_{\text {syst }}\right) \mathrm{cpd} / 100$ ton, corresponding to an equivalent flux $\Phi_{8_{B}}=(2.4 \pm 0.4 \pm 0.1) \cdot 10^{6} \mathrm{~cm}^{-2} \mathrm{~s}^{-1}$ which, taking into account neutrino oscillations, corresponds to $(0.88 \pm 0.19)$ of the SSM prediction.

\section{pep and CNO neutrinos}

The pep neutrino energy of $1.44 \mathrm{MeV}$ lies at the boundary between the vacuum and the transition region of the electron neutrino survival probability. The pep neutrino flux is tightly related to the fundamental pp neutrino flux and is thus theoretically well constrained. Measuring the pep neutrino flux thus tests the fundaments of the Solar Model. In the same energy region lie neutrinos from the CNO cycle. CNO neutrinos are poorly constrained by the SSM and have never been dierectly detected so far. The observed pep neutrino rate in Borexino is $\left(3.13 \pm 0.23_{\text {stat }} \pm 0.23_{\text {syst }}\right) \mathrm{cpd} / 100$ ton, from which the corresponding flux can be calculated, assuming the current MSW-LMA parameters, as $\Phi_{\text {pep }}=(1.6 \pm 0.3) \cdot 10^{8} \mathrm{~cm}^{-2} \mathrm{~s}^{-1}$, corresponding to $(1.1 \pm 0.2)$ of the SSM predicted flux [8]. The same analysis, keeping the pep flux fixed at the SSM value, leads to an upper limit on the CNO flux: $\Phi_{C N O} \leq 7.4 \cdot 10^{8} \mathrm{~cm}^{-2} \mathrm{~s}^{-1}$, corresponding to a ratio of less than 1.4 of the SSM prediction [8].

\section{pp neutrinos}

Shortly after this conference the Borexino collaboration has released the first direct measurement of the solar pp neutrinos [9], and thus confirmed the fundament of the Standard Solar Model. The solar pp neutrino interaction rate measured by Borexino is $\left(144 \pm 13_{\text {stat }} \pm 10_{\text {syst }}\right) \mathrm{cpd} / 100 \mathrm{t}$ corresponding to a solar pp neutrino flux of $(6.6 \pm 0.7) \cdot 10^{10} \mathrm{~cm}^{-2} \mathrm{~s}^{-1}$ (including neutrino oscillations). This value is in good agreement with the SSM predictions. The measured flux can also be used to derive the total energy radiated by the Sun, $3.84 \cdot 10^{33} \mathrm{erg} \mathrm{s}^{-1}$ demonstrating that about $99 \%$ of the energy of the Sun is really generated by the pp fusion process. Finally, the survival probability for pp neutrinos produced in the core of the Sun is $P_{e e}=0.64 \pm 0.12$, in agreement with the MSW-LMA solution in the low-energy vacuum regime (see fig.3).

\section{Conclusion}

The solar neutrino fluxes measured in real-time by Borexino and by the Cherenkov experiments SNO and Super-Kamiokande are in good agreement with the SSM predictions but are currently unable to discriminate between high and low metallicity models, due to the experimental 


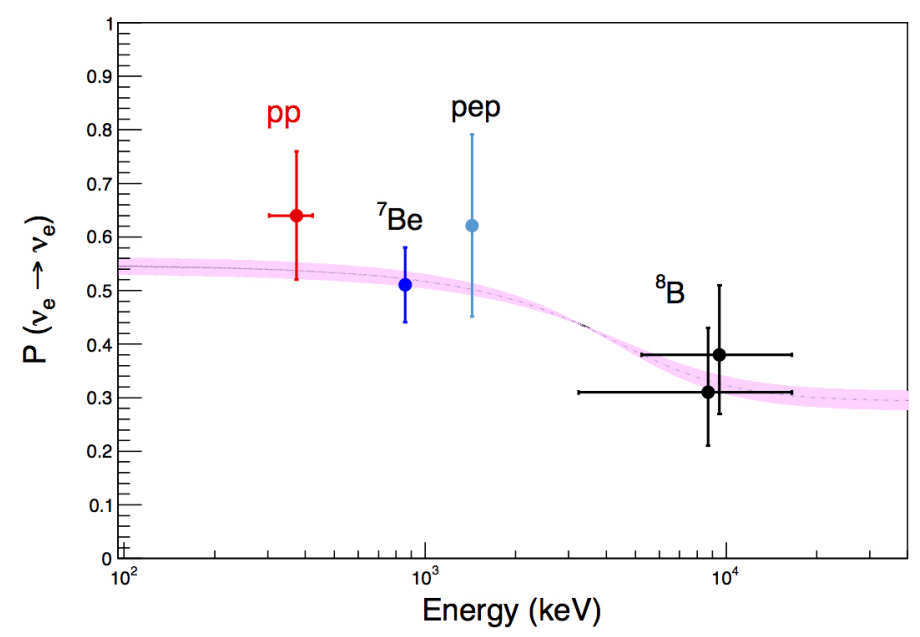

Figure 3: The predicted electron neutrino survival probability for the best-fit LMA solution together with the measured values for the individual neutrino branches as measured by Borexino. Taken from [9].

errors as well as uncertainties in the solar model predictions. A better accuracy of the measured neutrino fluxes will help to discriminate between the high and low metallicity solar models only if also the theoretical uncertainties on the predicted neutrino fluxes can be significantly reduced. With the first direct determination of the pp neutrinos Borexino has achieved an independent measurement of the neutrino luminosity of the Sun at the $10 \%$ level, proving that the Sun is in a stable hydrogen-burning state.

On the particle physics side, the low threshold measurements of the ${ }^{8} \mathrm{~B}$ neutrino flux by SNO and Super-Kamiokande leave open the question whether the electron neutrino survival probability follows the prediction of the LMA-MSW solution in the few MeV range. More statistics and a possible lower threshold measurement performed by Super-Kamiokande will help to clarify the situation in the near future.

\section{References}

[1] J. N. Bahcall, Phys. Rev. Lett. 12, 300 (1964)

[2] R. Davis, Jr., Phys. Rev. Lett. 12, 303 (1964)

[3] J. Beringer et al. (Particle Data Group), Phys. Rev. D86, 010001 (2012)

[4] B. Aharmim et al. (SNO collaboration), Phys. Rev. C88, 025501 (2013)

[5] A. Renshaw et al. (Super-Kamiokande collaboration), Phys. Rev. Lett. 112, 091805 (2014)

[6] A. Renshaw et al. (Super-Kamiokande collaboration), TAUP 2013 proceedings, arXiv:1403.4575 (2014)

[7] G. Bellini et al. (Borexino collaboration), Phys. Rev. D 89, 112007 (2014)

[8] G. Bellini et al. (Borexino collaboration), Phys. Rev. Lett. 108, 051302 (2012)

[9] G. Bellini et al. (Borexino collaboration), Nature 512, 7515, 383 (2014) 